Marquette University

e-Publications@Marquette

$10-23-2014$

\title{
Structure, Stability, and Substituent Effects in Aromatic S-Nitrosothiols: The Crucial Effect of a Cascading Negative Hyperconjugation/Conjugation Interaction
}

Matthew Flister

Marquette University, matthew.flister@marquette.edu

Qadir K. Timerghazin

Marquette University, qadir.timerghazin@marquette.edu

Follow this and additional works at: https://epublications.marquette.edu/chem_fac

Part of the Chemistry Commons

\section{Recommended Citation}

Flister, Matthew and Timerghazin, Qadir K., "Structure, Stability, and Substituent Effects in Aromatic $S$-Nitrosothiols: The Crucial Effect of a Cascading Negative Hyperconjugation/Conjugation Interaction" (2014). Chemistry Faculty Research and Publications. 360.

https://epublications.marquette.edu/chem_fac/360 
Marquette University

e-Publications@Marquette

\section{Chemistry Faculty Research and Publications/College of Arts and Sciences}

This paper is NOT THE PUBLISHED VERSION; but the author's final, peer-reviewed manuscript. The published version may be accessed by following the link in the citation below.

Journal of Physical Chemistry : A, Vol. 18, No. 42 (October 23, 2014): 9914-9924. DOI. This article is () American Chemical Society Publications and permission has been granted for this version to appear in e-Publications@Marquette. American Chemical Society Publications does not grant permission for this article to be further copied/distributed or hosted elsewhere without the express permission from American Chemical Society Publications.

\section{Structure, Stability, and Substituent Effects in Aromatic S-Nitrosothiols: The Crucial Effect of a Cascading Negative Hyperconjugation/Conjugation Interaction}

Matthew Flister

Department of Chemistry, Marquette University, Milwaukee, Wisconsin

Qadir K. Timerghazin

Department of Chemistry, Marquette University, Milwaukee, Wisconsin 


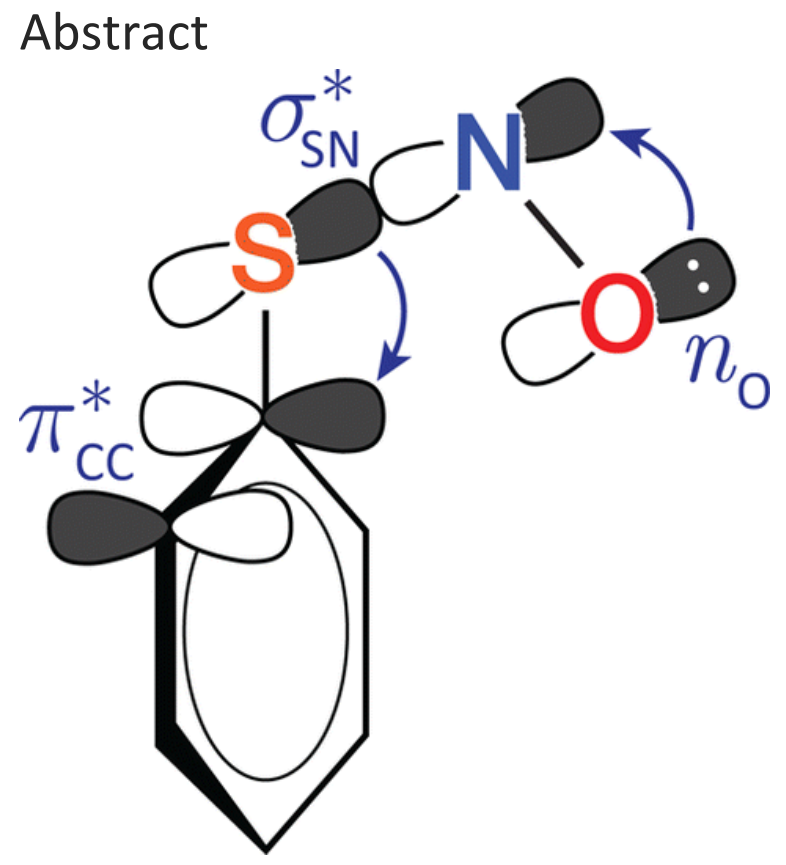

Aromatic S-nitrosothiols (RSNOs) are of significant interest as potential donors of nitric oxide and related biologically active molecules. Here, we address a number of poorly understood properties of these species via a detailed density functional theory and the natural bond orbital (NBO) investigation of the parent PhSNO molecule. We find that the characteristic perpendicular orientation of the -SNO group relative to the phenyl ring is determined by a combination of the steric factors and the donor-acceptor interactions including, in particular, a cascading orbital interaction involving electron delocalization from the oxygen lone pair to the $\sigma$-antibonding $\mathrm{S}-\mathrm{N}$ orbital and then to the $\pi^{*}$-aromatic orbitals, an unusual negative hyperconjugation/conjugation long-range delocalization pattern. These interactions, which are also responsible for the relative weakness of the $\mathrm{S}-\mathrm{N}$ bond in PhSNO and the modulation of -SNO group properties in substituted aromatic RSNOs, can be interpreted as a resonance stabilization of the ionic resonance component $\mathrm{RS}^{-} / \mathrm{NO}^{+}$of the RSNO electronic structure by the aromatic ring, similar to the resonance stabilization of $\mathrm{PhS}^{-}$anion. These insights into the chemistry and structure-property relationships in aromatic RSNOs can provide an important theoretical foundation for rational design of new RSNOs for biomedical applications.

\section{Introduction}

$S$-Nitrosothiols or thionitrites (RSNOs) have recently emerged as major players in the nitric oxide regulated biochemical processes.(1-4) Endogenous cysteine-based RSNOs not only act as a pool for NO storage and transport: in fact, tightly regulated $S$-nitrosation of select cysteine residues in proteins is also one of the major mechanisms involved in the biological function of nitric oxide. $(3,5-7)$ Cellular and extracellular RSNOs can be efficient $\mathrm{NO}$ or $\mathrm{NO}^{+}$donors via homolysis and trans-S-nitrosation reactions, respectively; they also may give rise to other biologically active nitrogen species such as nitroxyl(8-11) $\mathrm{HNO} \mathrm{NO}^{-}$or thionitrous acid $\mathrm{HSNO}$, itself the smallest, inorganic RSNO.(12-14)

Currently, there is a significant interest in therapeutic applications of naturally occurring (e.g., $S$ nitrosoglutathione) or synthetic RSNOs.(11, 15-21) By modifying the chemical nature of the substituent R, RSNOs can be potentially fine-tuned to efficiently release NO, act as specific trans-S-nitrosation reagents, or, possibly, as biologically compatible $\mathrm{HNO} / \mathrm{NO}^{-}$donors. To this end, a fundamental chemical understanding of RSNO structure, stability, and chemical properties is essential. 
Due to its unusual electronic structure, the -SNO group lends itself to unprecedented modulation either by the environment or substituent effects. The hallmark of RSNOs is the unusually elongated $(\sim 1.8 \AA)$ and weak S-N bond with bond dissociation energy, BDE, $\sim 30 \mathrm{kcal} / \mathrm{mol} .(22-26)$ At the same time, RSNOs exist in either cis or trans conformations indicative of partial double-bond character of the $\mathrm{S}-\mathrm{N}$ linkage. $(27,28)$ The $\mathrm{S}-\mathrm{N}$ bond properties in RSNOs can be significantly modified by interactions with Lewis acids that either stabilize or further destabilize it, depending which atom of the -SNO group the Lewis acid coordinates to.(29-33) Moreover, RSNOs demonstrate a dual-mode reactivity toward nucleophiles that can attack the -SNO group either at N or S atoms. $(33,34)$ These interesting and often contradictory properties of RSNOs stem from the complex and unique electronic structure of the -SNO group that can be reconciled using an elegant resonance representation (Chart 1).(35)

This resonance description features, in addition to the traditional Lewis structure with single $\mathrm{S}-\mathrm{N}$ bond, $\boldsymbol{S}$, two additional structures, a zwitterionic structure $\boldsymbol{D}$ and a no-bond ionic structure $\boldsymbol{I}$. The contribution of these two antagonistic resonance structures,(36) i.e., structures that imply opposite bonding patterns and formal charges, rationalizes the contradictory properties of the $\mathrm{S}-\mathrm{N}$ bond and their modulation by a Lewis acid coordination, $(33,35,36)$ as well as the dual-mode reactivity of RSNOs. $(34,37)$ Indeed, structure $\boldsymbol{D}$ accounts for the cis-trans isomerism and the $S$ atom directed reactions with nucleophiles, while structure $I$ explains the elongated, weak $\mathrm{S}-\mathrm{N}$ bond and $\mathrm{N}$-directed reactions with nucleophiles. The complexity of the RSNO electronic structure highlighted by this description also correlates with the modest multireference character of the -SNO group. $(38,39)$

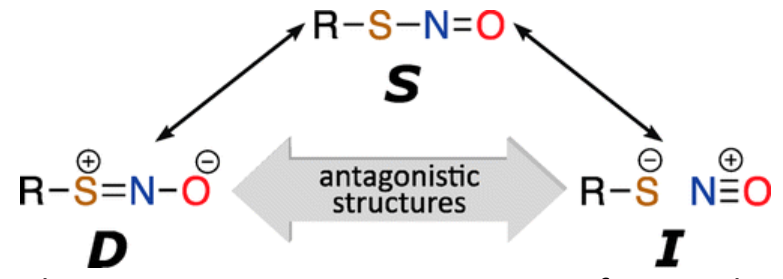

Chart 1. Resonance Representation of RSNO Electronic Structure

RSNO interactions with charged and neutral Lewis acids and bases or external electric fields can dramatically shift the $\boldsymbol{D}$ vs I balance and thus modulate the RSNO properties and reactivity.(34) This way, the S-N bond length and dissociation energy can be tuned within a wide range, 1.6-2.2 $\AA$ and $28-40 \mathrm{kcal} / \mathrm{mol}$, respectively, and various RSNO reactions can be catalyzed or inhibited.(34-37) Modulation of the -SNO group stability and reactivity by charged residues in proteins may form a basis for enzymatic control of RSNO reactions in vivo.(36) One may expect that similar modulation can be achieved by modification of the electrondonating/withdrawing properties of the substituent $R$ that should also affect the $\boldsymbol{D}$ vs $\boldsymbol{I}$ balance, which opens many avenues for rational design of RSNOs with desired chemical and biological properties. In this respect, aromatic RSNOs (ArSNOs) can provide a flexible template for creation of a wide range of novel RSNOs with desired stability and chemical reactivity.

Several ArSNOs have been reported in the literature, including the parent S-nitrosothiophenol PhSNO;(4046) they are generally less stable than aliphatic RSNOs, with half-lives less than $1 \mathrm{~h}$ at room temperature,(46) although ArSNOs with bulky substituents have been reported to be more stable.(43-45) A weak substituent effect has been observed with electron-donating substituents in para position having slightly longer half-lives.(46) A computational study by Marazzi et al. reported a significant substituent effect on the energy of ArSNO photoexcitation leading to homolytic $\mathrm{S}-\mathrm{N}$ bond cleavage.(47) Substituents in the para position were found to affect energies of the ${ }^{1}\left(n, \pi^{*}\right)$ and ${ }^{1}\left(\pi, \pi^{*}\right)$ transitions of the -SNO group; the latter transition is more sensitive to the substituent nature and decreases proportionally to the electron-withdrawing nature of the 
para substituent. Conversely, the ${ }^{1}\left(n, \pi^{*}\right)$ excitation energy increases proportionally with the electronwithdrawing power of the para substituent.

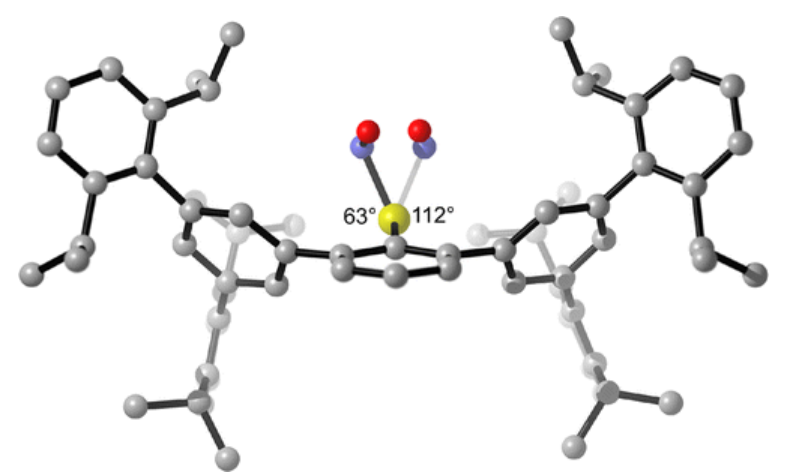

Figure 1. Experimental X-ray structure of an aromatic RSNO stabilized by bulky substituents at ortho positions reported by Goto et al.(43) Both possible positions of the disodered NO group are shown, along with the corresponding $\mathrm{C}-\mathrm{C}-\mathrm{S}-\mathrm{N}$ dihedral angle values.

This modulation of the -SNO group properties in ArSNOs is rather surprising, as there is no apparent $\pi$ conjugation between the aromatic ring and the -SNO group. Indeed, all ArSNO geometries reported by Marazzi et al. have the -SNO group noncoplanar with the aromatic ring. In cis-PhSNO, the -SNO group is almost perpendicular with the aromatic ring, with the $\mathrm{C}-\mathrm{C}-\mathrm{S}-\mathrm{N}$ dihedral angle $\sim 90^{\circ}$, while for other cis-ArSNOs this angle is reported to vary from $90^{\circ}$ to $70^{\circ}$. The trans-ArSNOs tend to have even more tilted -SNO group with C$\mathrm{C}-\mathrm{S}-\mathrm{N}$ dihedral in the $85^{\circ}-50^{\circ}$ range. Similar noncoplanar geometries with the $-\mathrm{SNO}$ group tilted toward the aromatic ring have been observed in experimental X-ray structures of cis-ArSNOs with bulky substituents at ortho-positions (Figure 1). $(43,44)$ In contrast to ArSNOs, $\pi$-conjugation between the $-S N O$ group and the $\mathrm{C}=\mathrm{C}$ double bond seems to be present in vinyl-subsitituted $\mathrm{RSNO}, \mathrm{CH}_{2}=\mathrm{CH}-\mathrm{SNO}$, predicted to have a planar geometry.(47)

At the moment, there is no satisfactory explanation for these interesting properties of ArSNOs. The factors that determine the instability of ArSNOs, noncoplanar orientation of the -SNO group, and the origins of the variability in its tilt relative to the aromatic ring are not clear. Moreover, the pronounced effect of para substituents in the absence of the SNO-phenyl ring $\pi$-conjugation is especially intriguing. Further progress with the rational design of ArSNOs, and RSNOs in general, is not possible without understanding these fundamental issues. Therefore, in this contribution we use electronic structure calculations and the natural bond orbital(48) (NBO) analysis to examine the electronic factors that determine the structure and stability of ArSNOs on the example of the parent PhSNO molecule compared with the well-studied aliphatic methyl-substituted RSNO, MeSNO.

\section{Computational Details}

All calculations have been performed using density functional theory (DFT) with a Perdew-Burke-Ernzerhof hybrid functional(49, 50) (PBE0), as implemented in the Gaussian 09 program package,(51) and the diffuseaugmented polarization-consistent aug-pc-1 basis set by Jensen.(52-54) We have shown previously(25, 36) that the PBEO functional provides a reliable description of the RSNO properties. Unless specified otherwise, the DFT calculations employed a pruned integration grid with 99 radial shells and 590 points per shell ("UltraFine" grid as defined in Gaussian 09). Geometry optimizations were performed in redundant internal coordinates(55) with "very tight" optimization criteria (i.e., $15 \times 10^{-6}$ and $10 \times 10^{-6}$ au for maximum and RMS force, respectively, $60 \times$ $10^{-6}$ and $40 \times 10^{-6}$ au for maximum and RMS displacement). Relaxed potential energy surface (PES) scans were performed by scanning along the specified dihedral angles. The conformational profiles along the $\mathrm{C}-\mathrm{S}$ bond in PhSNO have been obtained by scanning along one of the $\mathrm{C}-\mathrm{C}-\mathrm{S}-\mathrm{N}$ angles; however, due to a slight 
pyramidalization of the $\mathrm{C}-\mathrm{S}$ carbon atom this dihedral angle does not provide an unambiguous measure of the -SNO group relative to the phenyl ring. Therefore, the resulting profiles have been plotted against an interplane angle $\varphi$ measured as an angle between the SNO and CCC planes, where the central carbon is bonded to the sulfur of the -SNO group. Natural bond orbital (NBO) family analyses(48) were performed with the latest version of NBO 6.0 code,(56) unless specified otherwise. Orbital interaction energies were estimated using second-order perturbation theory, and the steric repulsion energies were estimated using natural steric analysis. $(48,57)$

\section{Results and Discussion}

\section{PhSNO vs MeSNO: Stability, Geometry, and Potential Energy Surfaces}

The $\mathrm{S}-\mathrm{N}$ bond in PhSNO is less stable relative to MeSNO, with calculated bond dissociation energy (BDE) 26.3 $\mathrm{kcal} / \mathrm{mol}$ vs $31.7 \mathrm{kcal} / \mathrm{mol}$ in MeSNO (both in cis conformations, Figure $2 \mathrm{~A}$ ), in agreement with the experimental observations that ArSNOs are generally less stable compared to alkyl-substituted RSNOs. $(23,45,46,58)$ Both molecules have similar conformational profiles for the $-\mathrm{NO}$ group rotation along the $\mathrm{S}-\mathrm{N}$ bond, with the cis conformer lower in energy by $\sim 1 \mathrm{kcal} / \mathrm{mol}$ in both cases (Figure $3 \mathrm{~A}$ ). The transition structures of cis-trans isomerization $\mathrm{TS}_{\mathrm{ct}}$ have similar geometries, although the isomerization barrier is $\sim 3 \mathrm{kcal} / \mathrm{mol}$ lower for PhSNO, $11.1 \mathrm{vs} 14.3 \mathrm{kcal} / \mathrm{mol}(10.5$ and $13.9 \mathrm{kcal} / \mathrm{mol}$ including ZPE). Independent of the $-\mathrm{NO}$ group rotation, the $\mathrm{S}-\mathrm{N}$ bond length in PhSNO is $\sim 0.06 \AA$ longer than in MeSNO, although the $\mathrm{N}-\mathrm{O}$ bond is slightly shorter by $\sim 0.01 \AA$ (Figure $2 \mathrm{~A})$. In both cases, the $\mathrm{S}-\mathrm{N}$ bond is highly elongated $(\sim 0.14 \AA)$ at the top of the cis-trans isomerization barrier $\mathrm{TS}_{\mathrm{ct}}\left(\mathrm{C}-\mathrm{S}-\mathrm{N}-\mathrm{O}\right.$ angle $\left.\sim 85-87^{\circ}\right)$, and in the case of PhSNO it becomes $>2 \AA$, well above the typical single $\mathrm{S}-$ $\mathrm{N}$ bond length $(\sim 1.6-1.7 \AA)$.(59)

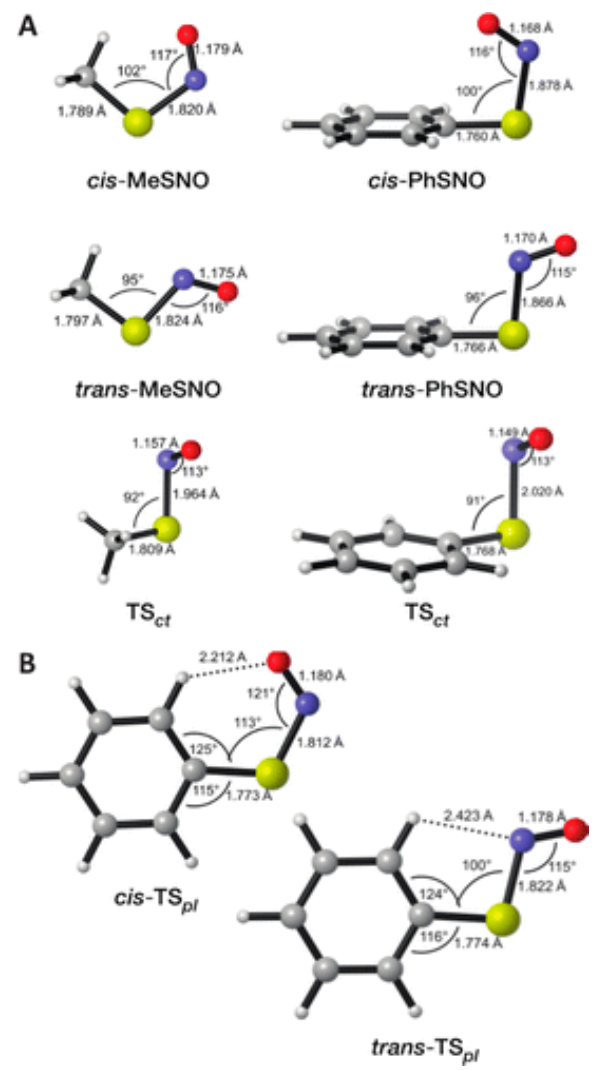

Figure 2. Calculated equilibrium structures of cis and trans conformers and the cis-trans isomerization transition structures $\mathrm{TS}_{\mathrm{ct}}$ for MeSNO and PhSNO (A). Coplanar structures of the two PhSNO conformers that correspond to transion structures for the rotation along the $\mathrm{C}-\mathrm{S}$ bond, $\mathrm{TS}_{\mathrm{pl}}(\mathrm{B})$. 
For both cis- and trans-PhSNO, the $C_{S}$ symmetric structures with the angle between $\varphi$ the $\mathrm{C}-\mathrm{S}-\mathrm{N}$ and $\mathrm{C}-\mathrm{C}-\mathrm{C}$ planes (Figure 4) exactly at $90^{\circ}$ appear as local minima points on the PES, without imaginary vibrational frequencies. However, geometry optimizations of trans-PhSNO without symmetry constraints using standard or "UltraFine" (as defined in Gaussian 09) DFT integration grids arrive at slightly bent structures with $\varphi=81^{\circ}-86^{\circ}$ essentially isoenergetic $(<0.02 \mathrm{kcal} / \mathrm{mol})$ with the $C_{S}$ structure. At the same time, calculations using more accurate grid settings ("SuperFine" in Gaussian 09 rev. D) yield perpendicular geometry with $\varphi=90^{\circ}$.
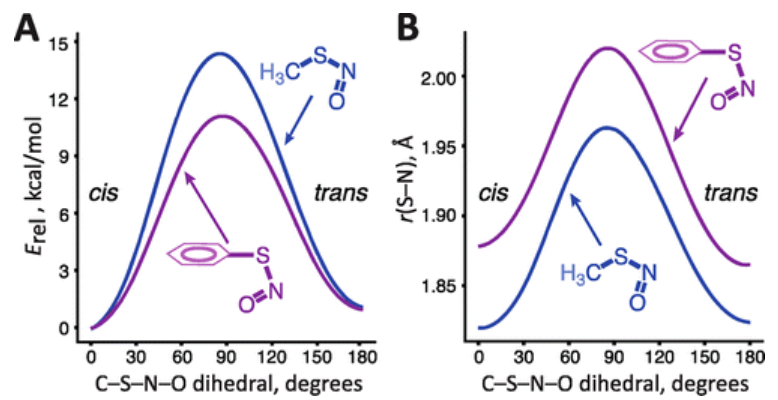

Figure 3. Relaxed potential energy profiles for rotation around the $\mathrm{C}-\mathrm{S}-\mathrm{N}-\mathrm{O}$ dihedral angle in $\mathrm{MeSNO}$ and PhSNO (A) and the corresponding evolution of the $\mathrm{S}-\mathrm{N}$ bond length (B).

The extreme flatness of the trans-PhSNO PES is further evident from the relaxed PES scan (Figure 4) that shows the energy change of $<0.1 \mathrm{kcal} / \mathrm{mol}$ within $\pm 30^{\circ}$ around the perpendicular structure. The cis-PhSNO PES is less flat around the minimum at $\varphi=90^{\circ}$; the energy gradually increases as the -SNO group twists toward the coplanar structure that corresponds to a first-order saddle point/transition structure $\mathrm{TS}_{\mathrm{pl}}$ (Figure $2 \mathrm{~B}$ ) with associated $5.3 \mathrm{kcal} / \mathrm{mol}$ energetic barrier $(4.8 \mathrm{kcal} / \mathrm{mol}$ with ZPE correction), which is $\sim 50 \%$ lower in the case of the trans conformer, $2.6 \mathrm{kcal} / \mathrm{mol}\left(2.2 \mathrm{kcal} / \mathrm{mol}\right.$ with ZPE). Both cis and trans coplanar structures $\mathrm{TS}_{\mathrm{pl}}$ have unequal and highly distorted $\mathrm{C}-\mathrm{C}-\mathrm{S}$ angles, $\sim 115^{\circ}$ and $\sim 125^{\circ}$ (Figure $2 \mathrm{~B}$ ), compared to symmetric perpendicular structures with $\sim 120^{\circ} \mathrm{C}-\mathrm{C}-\mathrm{S}$ angles.

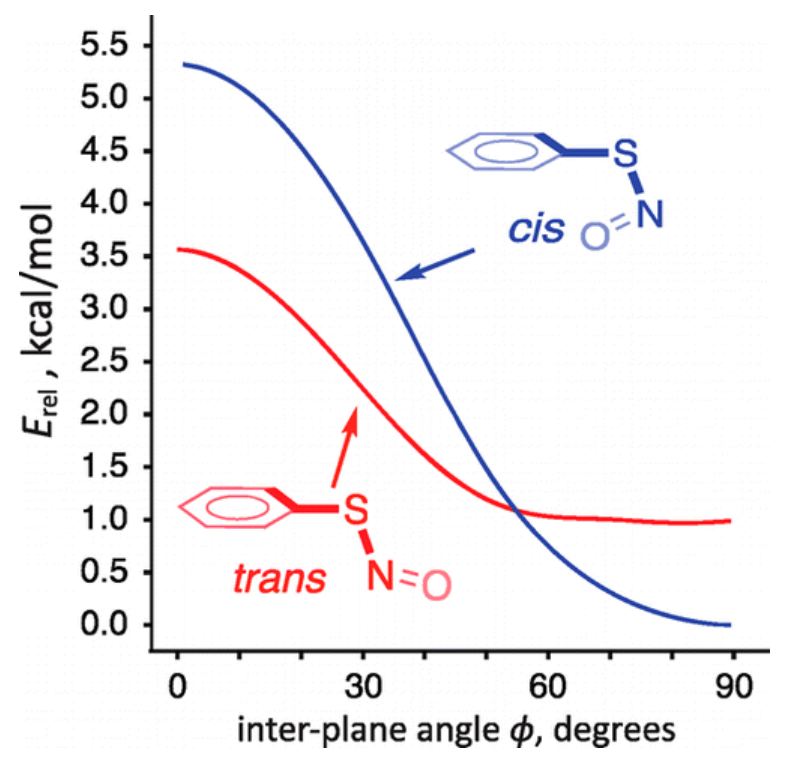

Figure 4. Relaxed potential energy profile for the -SNO group rotation along the $\mathrm{C}-\mathrm{S}$ bond in terms of an angle between the $\mathrm{C}-\mathrm{S}-\mathrm{N}$ and $\mathrm{C}-\mathrm{C}-\mathrm{C}$ planes, $\varphi$, which is used instead of the $\mathrm{C}-\mathrm{C}-\mathrm{S}-\mathrm{N}$ dihedral angle due to a slight pyramidalization at the $\mathrm{C}-\mathrm{S}$ carbon atom.

The -SNO group tilting angle $\varphi$ strongly affects the $\mathrm{S}-\mathrm{N}$ and $\mathrm{C}-\mathrm{S}$ bond lengths in PhSNO. The $\mathrm{S}-\mathrm{N}$ bond is longest for the minimum energy structures with $\varphi=90^{\circ}$ and shortest for the coplanar structures $\mathrm{TS}_{\mathrm{pl}}$ with $\varphi=0^{\circ}$ (Figure S1A in the Supporting Information). Similarly to the energy profiles (Figure 4), the S-N bond length profiles are 
relatively flat around the minimum energy structures, $\varphi=90^{\circ} \pm 30^{\circ}$. The C $-\mathrm{S}$ bond initially shortens as the $-\mathrm{SNO}$ group tilts toward coplanarity with the aromatic ring, up to $\varphi=90^{\circ} \pm\left(30^{\circ}-40^{\circ}\right)$, and then quickly lengthens and reaches its maximum length for the coplanar structure, Figure S1B in the Supporting Information. Thus, the S-N bond length and, likely, its strength anticorrelates with the overall energetic stability of PhSNO. On the other hand, there is a correlation between the $\mathrm{C}-\mathrm{S}$ bond length and the overall stability of PhSNO. This pronounced dependence of PhSNO energy and geometry on the relative orientation of the -SNO group and the aromatic ring suggests that there is a strong interaction between the two moieties. As we demonstrate below, this interaction arises from a complex combination of electronic effects including steric/Pauli repulsion between filled orbitals and conjugative donor-acceptor/charge-transfer delocalization of electron density from filled donor orbitals to formally unoccupied acceptor orbitals of the -SNO group and the phenyl ring.

\section{PhSNO vs MeSNO: Conjugative and Steric Effects within the -SNO Group}

The conjugative donor-acceptor orbital interactions that determine the unusual properties of the -SNO group have been extensively studied on the example of MeSNO using the NBO approach.(35, 36, 60) The two major orbital interactions shown in Figure S2A in the Supporting Information, the $\pi$-conjugation between the lone pair of sulfur $n_{\mathrm{S}}$ and the antibonding $\pi_{\mathrm{NO}}{ }^{*}$ orbital, $n_{\mathrm{S}} \rightarrow \pi_{\mathrm{NO}}{ }^{*}$, and the negative hyperconjugation between the lone pair of oxygen $n_{O}$ and the antibonding $\sigma_{S N}{ }^{*}$ orbital, $n_{O} \rightarrow \sigma_{S N}{ }^{*}$, determine the rotational barrier along the $S-N$ bond and the elongation/weakness of that bond, respectively. These two orbital interactions are also the electronic basis of the antagonistic RSNO resonance structures $\boldsymbol{D}$ and $\boldsymbol{I}$, respectively (Chart 1 ).

Conjugative interactions that require overlap between an occupied orbital with an unoccupied antibonding orbital also mean that the donor orbital should overlap with the corresponding bonding orbital, thus resulting in Pauli/steric repulsion. In MeSNO, the energetic effect of these $\left.n_{s}\right)\left(\pi_{\mathrm{NO}}\right.$ repulsions (using the notation from ref 61) evaluated with the natural steric analysis(57) are relatively large, $\sim 6$ and $\sim 30 \mathrm{kcal} / \mathrm{mol}$, respectively (Figure S2B in the Supporting Information). The estimates of the stabilizing $n_{\mathrm{S}} \rightarrow \pi_{\mathrm{NO}^{\circ}}{ }^{*}$ and $n_{\mathrm{O}} \rightarrow \sigma_{\mathrm{SN}}{ }^{*}$ donor-acceptor interactions obtained with the second-order perturbation theory, $\Delta E_{i j}^{(2)} \approx 25$ and $52-58 \mathrm{kcal} / \mathrm{mol}$, respectively, are clearly stronger than the corresponding steric repulsions (Figure S2, A and B in the Supporting Information). Although the donor-acceptor delocalization energies $\Delta E_{i j}^{(2)}$ and the steric repulsion energies $\Delta E_{i j}{ }^{(\text {sx) }}$ are evaluated using different sets of orbitals, NBO and NLMO, respectively, and therefore may not be directly compatible, the overall stabilization effect due to the donor-acceptor interactions is clearly evident.

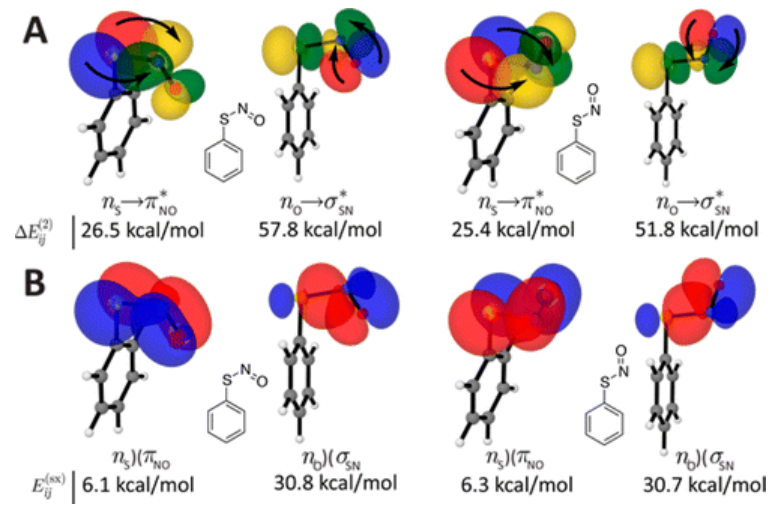

Figure 5. Dominant stabilizing donor-acceptor orbital interactions (A) and destabilizing steric repulsions (B) in cis- and trans-PhSNO shown using isosurface representations of the preorthogonal natural bond orbitals (PNBOs, A) and preorthogonal natural localized molecular orbitals (PNLMOs, B). Note that although the respective energetic effects estimated with the second-order perturbation theory, $\Delta E_{i j}{ }^{(2)}$, and natural steric analysis, $\Delta E_{i j}{ }^{(s x)}$ are given as positive numbers, they have opposite energetic effects on the stability of the molecule. 
The donor-acceptor stabilizations and steric repulsions within the -SNO group in PhSNO (Figure 5) are qualitatively identical to MeSNO (Figure S2 in the Supporting Information). However, the negative hyperconjugation $n_{\mathrm{O}} \rightarrow \sigma_{\mathrm{SN}}{ }^{*}$ is stronger in PhSNO (by 10-20 kcal/mol), whereas the $\pi$-conjugation $n_{\mathrm{S}} \rightarrow \pi_{\mathrm{NO}}{ }^{*}$ is weaker (by 4-7 kcal/mol), which correlates with longer and weaker S-N bond in PhSNO, as well as with lower cis-trans isomerization barrier (Figure $3 \mathrm{~A})$. Similarly, the steric repulsions $\left.n_{S}\right)\left(\pi_{N O}\right.$ and $\left.n_{O}\right)$ ( $\sigma_{S N}$ are slightly weaker and stronger, respectively. However, the origin of the increased ionic character of the $\mathrm{S}-\mathrm{N}$ bond (represented by the resonance structure $I$, Chart 1 ) signified by the increased $n_{\mathrm{O}} \rightarrow \sigma_{\mathrm{SN}}{ }^{*}$ negative hyperconjugation and decreased $n_{\mathrm{S}} \rightarrow \pi_{\mathrm{NO}}{ }^{*}$ conjugation can be understood only by examining the electronic interactions between the -SNO group and the aromatic $\pi$ system.

\section{SNO-Aromatic Ring Steric Repulsion}

The general shape of the PhSNO PES with respect to the -SNO group rotation relative to the aromatic ring (Figure 4) can be quantitatively rationalized by Pauli/steric repulsions between the two moieties (Figure 6). The maxima that determine the noncoplanar geometry of cis- and trans-PhSNO arise from the steric clash between the $-\mathrm{SNO}$ group and the adjacent $\mathrm{C}-\mathrm{H}$ bonds, which results in contorted geometries with distorted $\mathrm{C}-\mathrm{C}-\mathrm{S}$ angles, $\sim 115^{\circ}$ and $\sim 125^{\circ}$ (Figure $2 \mathrm{~B}$ ), that minimize the steric repulsions at the price of the energetic cost associated with the distortion. The residual steric $-\mathrm{SNO})(\mathrm{C}-\mathrm{H}$ repulsions calculated with the natural steric analysis thus account only in part for the barrier height corresponding to the coplanar structure. In cis-PhSNO (Figure 4 ) the $\left.n_{\mathrm{O}}\right)$ ( $\sigma_{\mathrm{CH}}$ repulsion between the two lone pairs of oxygen, predominantly of $s$ and $p$ character, respectively, and the $\sigma_{\mathrm{CH}}$ orbital has the overall energy of $5.2 \mathrm{kcal} / \mathrm{mol}$ (Figure $6 \mathrm{~A}$ ). The steric clash is less pronounced in trans-PhSNO, with weaker $\left.n_{\mathrm{N}}\right)\left(\sigma_{\mathrm{CH}}\right.$ repulsion between the nitrogen lone pair and the $\sigma_{\mathrm{CH}}$ orbital, $3.5 \mathrm{kcal} / \mathrm{mol}$, Figure $6 \mathrm{~A}$, which correlates with $\sim 2.5 \mathrm{kcal} / \mathrm{mol}$ lower barrier for the rotation along the $\mathrm{C}-\mathrm{S}$ bond in this conformer (Figure 4). Both $\left.n_{\mathrm{O}}\right)\left(\sigma_{\mathrm{CH}}\right.$ and $\left.n_{\mathrm{N}}\right)\left(\sigma_{\mathrm{CH}}\right.$ repulsions quickly disappear as the -SNO group rotates out of coplanarity with the phenyl ring.

However, the steric repulsions alone do not seem to be able to rationalize the PhSNO preference toward the perpendicular SNO-aromatic ring arrangement. Indeed, the minimum-energy perpendicular structures also result in several steric repulsions between the occupied orbitals of the -SNO and phenyl moieties, including relatively strong $(\sim 6.5-8 \mathrm{kcal} / \mathrm{mol})$ interactions arising from the overlap of the $\mathrm{S}-\mathrm{N}$ bond with the aromatic $\pi$ system, $\left.\sigma_{S N}\right)\left(\pi_{c c}\right.$, which are in antiphase to weaker $\left.\sigma_{S N}\right)\left(\sigma_{c c}\right.$ repulsions in the coplanar structures $(\sim 4 \mathrm{kcal} / \mathrm{mol})$. For the trans conformer, the perpendicular structure also leads to repulsion between the nitrogen lone pair $n_{N}$ and the $\pi$-system, $\left.n_{N}\right)\left(\pi_{c c},(\sim 2 \mathrm{kcal} / \mathrm{mol})\right.$. Furthermore, a bulky $p$-type lone pair of sulfur $n_{\mathrm{S}}$ overlaps with the $\mathrm{C}-\mathrm{H}$ bonds, resulting in $\left.\sim 3 \mathrm{kcal} / \mathrm{mol} n_{S}\right)\left(\sigma_{\mathrm{CH}}\right.$ steric repulsion. Although the repulsions between the same $n_{\mathrm{s}}$ lone pair and the $\mathrm{C}-\mathrm{C} \sigma$ - and $\pi$-bonds, $\left.n_{\mathrm{s}}\right)\left(\sigma_{\mathrm{cc}}\right.$ and $\left.n_{\mathrm{s}}\right)\left(\pi_{\mathrm{cc}}\right.$, are significantly stronger, they are equal in strength and in antiphase to each other, resulting in a nearly constant net steric repulsion $\sim 10 \mathrm{kcal} / \mathrm{mol}$ that should not affect the PhSNO conformational profile (Figure 4).

Overall, the steric considerations alone seem to suggest that PhSNO should adopt a bent minimum energy strucure that avoids both strong $-\mathrm{SNO})(\mathrm{C}-\mathrm{H}$ steric clashes of the coplanar structures as well as weaker steric repulsions observed for the perpendicular structures. Therefore, there should be some stabilizing electronic factors that prefer perpendicular orientation of the -SNO group toward the aromatic ring.

\section{SNO-Aromatic Ring Donor-Acceptor Orbital Interactions}

NBO analysis of the stabilizing donor-acceptor orbital interactions between the -SNO and the phenyl group shows a number of interactions that mirror most of the steric repulsions discussed above. However, as the stabilizing energetic effect of donor-acceptor orbital interactions depends not only on the overlap between the interacting orbitals but also on the energy difference between them, there are some important differences 
between the orbital interaction energy profiles (Figure 7) and the corresponding steric repulsion energy profiles (Figure 6).
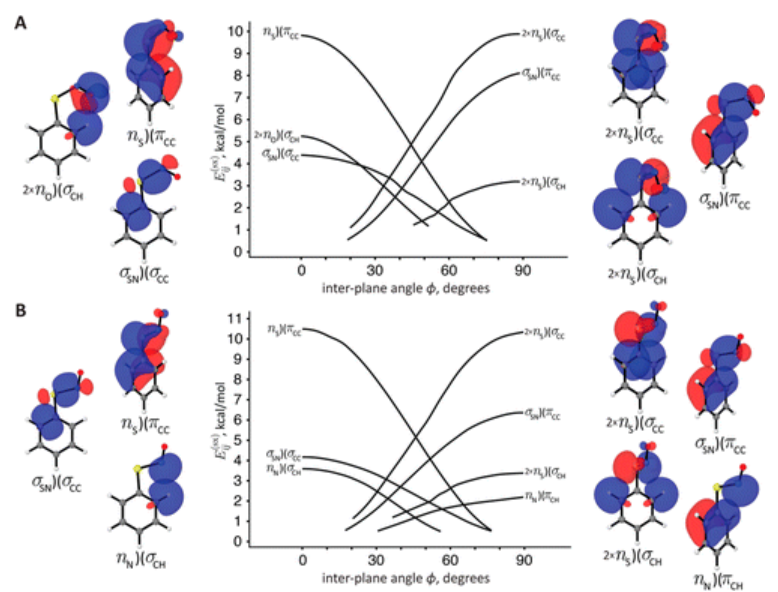

Figure 6. Major steric repulsions between the -SNO group and the phenyl ring in cis-PhSNO $(\mathrm{A})$ and trans-PhSNO (B) corresponding to the coplanar $\mathrm{TS}_{\mathrm{pl}}\left(\varphi=0^{\circ}\right.$, left $)$ and minimum energy perpendicular $\left(\varphi=90^{\circ}\right.$, right) structures, and the evolution of their energies with respect to the -SNO group rotation along the $\mathrm{C}-\mathrm{S}$ bond.

While the sulfur $p$-type lone pair $n_{\mathrm{s}}$ repulsions with occupied $\sigma_{\mathrm{cc}}$ and $\pi_{\mathrm{cc}}$ orbitals (in perpendicular and coplanar structures, respectively) are virtually equivalent in energy, the stabilizing effect of the $n_{\mathrm{S}} \rightarrow \pi_{c c}{ }^{*}$ interaction in the coplanar structure is much stronger compared to the $n_{\mathrm{S}} \rightarrow \sigma_{\mathrm{cc}}{ }^{*}$ interaction in the perpendicular structure, $\sim 19$ vs $10-11 \mathrm{kcal} / \mathrm{mol}$, respectively. In the perpendicular structure, the $\left.\sigma_{\mathrm{SN}}\right)\left(\pi_{\mathrm{cc}}\right.$ repulsion is mirrored by simultaneous $\pi_{c c}{ }^{*} \rightarrow \sigma_{S N}$ and $\sigma_{S N} \rightarrow \pi_{c c}{ }^{*}$ stabilizing interactions, 4-5 and 7-8 kcal/mol, respectively. Similarly, in the coplanar structure, the $\left.\sigma_{S N}\right)\left(\sigma_{C C}\right.$ repulsion is mirrored by a weak $\sigma_{S N} \rightarrow \sigma_{C C}{ }^{*}$ delocalization, $\sim 2 \mathrm{kcal} / \mathrm{mol}$. The $\left.n_{\mathrm{O}}\right)\left(\sigma_{\mathrm{CH}}, n_{\mathrm{N}}\right)\left(\sigma_{\mathrm{CN}}, n_{\mathrm{S}}\right)\left(\sigma_{\mathrm{CN}}\right.$, and $\left.n_{\mathrm{N}}\right)\left(\pi_{\mathrm{CC}}\right.$ steric repulsions do not have appreciably strong stabilizing conjugative equivalents.

The interplay of the destabilizing steric and stabilizing conjugative interactions between the -SNO and phenyl moieties that involve the $\sigma_{S N} / \sigma_{S N}{ }^{*}, n_{S}, \pi_{C C} / \pi_{C C}{ }^{*}$, and $\sigma_{c c} / \sigma_{C C}{ }^{*}$ orbitals can, in principle, account for the flatness of the trans-PhSNO conformational profile around the prerpendicular structure, $\varphi=0^{\circ}-60^{\circ}$. Another contribution to the PES flatness in this region could come from the $n_{\mathrm{s}} \rightarrow \pi_{c c}{ }^{*}$ delocalization that has an early onset, around $\varphi$ $=80^{\circ}$, and quickly rises in energy as the -SNO group moves toward coplanarity with the phenyl ring, thereby compensating the decreasing stabilization energy due to the $n_{S} \rightarrow \pi_{\subset C}{ }^{*}$ and $\pi_{C C} \rightarrow \sigma_{S N}{ }^{*} / \sigma_{S N} \rightarrow \pi_{C C}{ }^{*}$ interactions. The latter pair of donor-acceptor interactions between the $\mathrm{S}-\mathrm{N}$ bond and the aromatic $\pi$-system also may be able to account for the phenyl ring influence on the -SNO group properties and their modulation by the substituents in aromatic RSNOs. However, it is not clear if these interactions are strong enough to confer rather significant destabilization of the $\mathrm{S}-\mathrm{N}$ bond in PhSNO compared to MeSNO. Also, the conformational profiles of steric repulsions and donor-acceptor interactions (Figures 6 and 7) are very similar for cis- and trans-PhSNO which is inconsistent with the significant differences between the two energy profiles around the perpendicular geometry, $\varphi=90^{\circ}-60^{\circ}$ (Figure 4). 

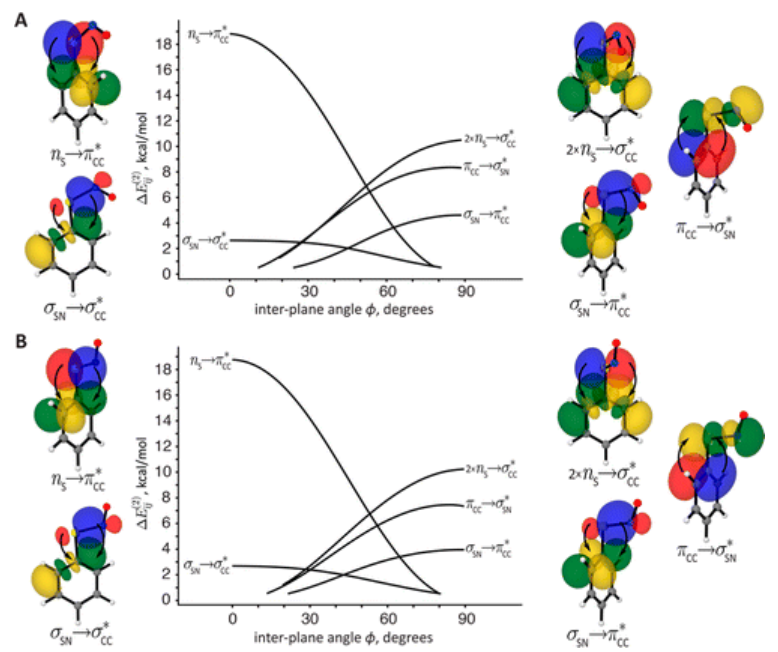

Figure 7. Major donor-acceptor interactions between the -SNO group and the phenyl ring orbitals in cis-PhSNO (A) and trans-PhSNO (B) corresponding to the coplanar $\mathrm{TS}_{\mathrm{pl}}\left(\varphi=0^{\circ}\right.$, left) and minimum energy perpendicular $(\varphi=$ $90^{\circ}$, right) strucutures, and the evolution of their energies with respect to the -SNO group rotation along the C$S$ bond (center).

These consideratons lead us to look for other possible factors involved in the intricate SNO-phenyl ring electronic communication. While the standard second-order perturbation theory analysis of the donor-acceptor interactions performed with recent versions of the NBO code (versions 5.9 and 6.0) does not reveal any additional interactions between the -SNO and phenyl moieties, the list of principal delocalizations generated by NBO 5.9 suggests, surprisingly, that the formally unoccupied antibonding $\sigma_{\mathrm{SN}}{ }^{*}$ orbital acts as a donor in a delocalization interaction with an adjacent $\pi$-antibonding orbital of the phenyl ring, $\pi_{\subset c}{ }^{*}$.(62)

Although somewhat unexpected, the existence of $\sigma_{\mathrm{SN}}{ }^{*} \rightarrow \pi_{\mathrm{CC}}{ }^{*}$ delocalization is in line with the electronic structure of the -SNO group, whose $\sigma_{S N}{ }^{*}$ orbital has a significant population ( 0.2 and $\sim 0.24 \mathrm{e}^{-}$in MeSNO and PhSNO, respectively) due to the strong $n_{O} \rightarrow \sigma_{S N}{ }^{*}$ negative hyperconjugation (Figures $6 \mathrm{~A}$ and $7 \mathrm{~A}$ ). Given the population and favorable alignment of the $\sigma_{S N}{ }^{*}$ orbital relative to the aromatic $\pi$-system in PhSNO with perpendicular -SNO group orientation (Figure 8), the secondary $\sigma_{S N}{ }^{*} \rightarrow \pi_{c c}{ }^{*}$ delocalization is not entirely surprising. This suggest that the energetic contribution of the $\sigma_{\mathrm{SN}}{ }^{*} \rightarrow \pi_{\mathrm{CC}}{ }^{*}$ interaction may play a significant role in determining the PhSNO PES and thus requires proper quantification.
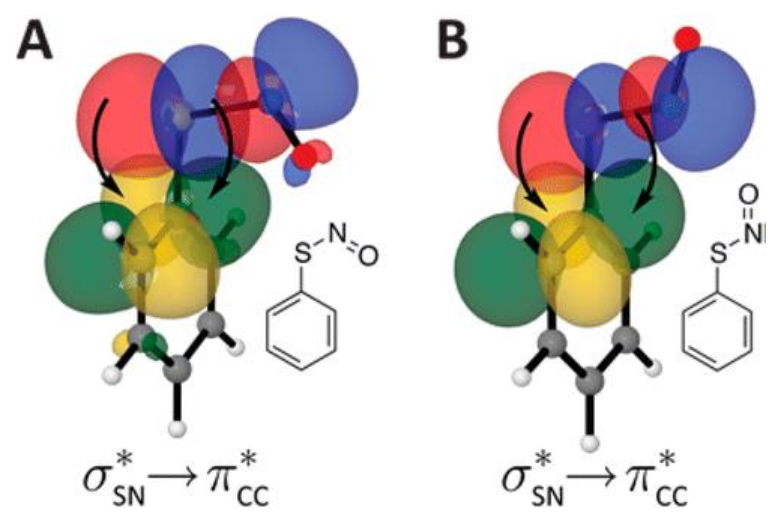

Figure 8. Donor-acceptor delocalization of electron density from the $\sigma_{S N}{ }^{*}$ orbital to a $\pi_{C C}{ }^{*}$ orbital of the aromatic ring in cis- $(\mathrm{A})$ and trans-PhSNO (B).

However, reliable estimation of the energetic effect of the $\sigma_{S N}{ }^{*} \rightarrow \pi_{\mathrm{cc}}{ }^{*}$ interaction is not straightforward methodologically. For instance, the second-order perturbation theory interaction energies $\Delta E_{i j}{ }^{(2)}$ are not meaningful in this case, because they are calculated assuming nondegenerate orbital energies for the donor (i) 
and acceptor ( $j$ ) orbitals: $\Delta E_{i j}^{(2)}=-q_{i} \frac{F_{i j}^{2}}{\Delta \varepsilon_{j i}}$ where $q_{i}$ is the donor orbital occupancy, $F_{i j}$ is the Fock matrix element, and $\Delta \varepsilon_{j i}$ is the difference between the acceptor and donor orbital energies, $\varepsilon_{j}$ and $\varepsilon_{i}$. As the $\sigma_{\mathrm{SN}}{ }^{*}$ and $\pi_{\subset C}{ }^{*}$ are very close in energy $\left(\Delta \varepsilon_{j i}<0.01\right.$ hartree) the second-order perturbation theory would give highly unphysical interaction energy estimates ( $>80 \mathrm{kcal} / \mathrm{mol})$.

Another approach to estimate the energetic effect of conjugative interactions in NBO is a quasi-variational deletion approach, when the donor-acceptor interaction of interest is deleted by removing the corresponding elements of the NBO Fock matrix, recalculating the density matrix and the corresponding electronic energy which is then compared to the full electronic energy of the molecule. The deletion technique suggests that the $\sigma_{\mathrm{SN}}{ }^{*} \rightarrow \pi_{\mathrm{CC}}{ }^{*}$ interaction exists in both PhSNO conformers (Figure 7), although its energetic effect is relatively small, $\triangle E(\$ D E L)=3.5-3.8 \mathrm{kcal} / \mathrm{mol}$. However, the $\triangle E(\$ D E L)$ values are also suspect, as the deletion technique may not be reliable when applied to the DFT densities.(48) It is recommended to confirm the $\triangle E(\$ D E L)$ values obtained with DFT by comparing them with the Hartree-Fock (HF) calculations. Unfortunately, the HF method provides an erroneous description of the basic -SNO group properties(38) and thus is not useful in the case of PhSNO. This prompted us to look for another approach to characterize the $\sigma_{\mathrm{SN}}{ }^{*} \rightarrow \pi_{\mathrm{CC}}{ }^{*}$ interaction using NBO methods.

\section{Characterization of Secondary Donor-Acceptor Interactions Using an Alternative Lewis}

\section{Structure}

To understand the chemical importance of the somewhat unconventional $\sigma_{\mathrm{SN}}{ }^{*} \rightarrow \pi_{c c}{ }^{*}$ interaction in PhSNO (Figure 8), we need to turn back to the basic resonance description of the -SNO group in terms of the conventional structure $\boldsymbol{S}$ and two antagonistic structures $\boldsymbol{D}$ and $\boldsymbol{I}$ (Chart 1 ). The latter structure represents the strong negative hyperconjugation $n_{O} \rightarrow \sigma_{S N}{ }^{*}$ (Figures 6A and 7A): when brought to completion, this interaction would result in breaking of the $\mathrm{S}-\mathrm{N} \sigma$-bond and formation of a second $\mathrm{N}-\mathrm{O} \pi$-bond, $\pi_{\mathrm{NO} 2}$, and a second $p$-type lone pair at the sulfur atom, $n_{\mathrm{S} 2}$. Thus, although the $\sigma_{\mathrm{SN}}{ }^{*} \rightarrow \pi_{\mathrm{CC}}{ }^{*}$ interactions cannot be reliably characterized using the default natural Lewis structure equivalent to the main resonance structure $S$, this secondary conjugation can be conveniently analyzed in terms of an alternative Lewis structure corresponding to the resonance structure $I$. This can be done using the \$CHOOSE option of the NBO program that expresses the electronic structure of a molecule in terms of a user-specified Lewis structure; the resulting NBO description corresponds to the same electron density, albeit expressed in terms of different orbital sets. For instance, the S$\mathrm{N}$ bond does not formally exist in the alternative $\$$ CHOOSE-I representation but appears, instead, as an extremlely strong $n_{\mathrm{S} 2} \rightarrow \pi_{\mathrm{NO} 2}{ }^{*}$ delocalization.
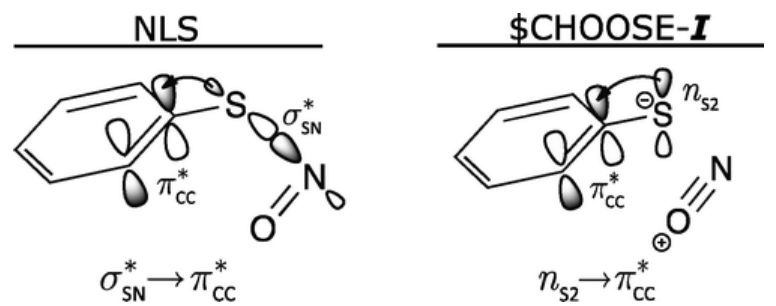

Chart 2. Secondary Conjugation in the Natural Lewis Structure versus Alternative \$CHOOSE-I Lewis Structure

In the $\$$ CHOOSE-I representation, the population of the $n_{\mathrm{S2}}$ lone pair corresponds to the sulfur atom portion of both $\sigma_{S N}$ and $\sigma_{S N}{ }^{*}$ orbital populations in the default natural Lewis structure $S$. Thus, both conventional $\sigma_{S N} \rightarrow$ $\pi_{\mathrm{CC}}{ }^{*}$ hyperconjugation (Figure 7) and unconventional secondary $\sigma_{\mathrm{SN}}{ }^{*} \rightarrow \pi_{\mathrm{CC}}{ }^{*}$ conjugation (Figure 8) are represented as a single $n_{\mathrm{S2}} \rightarrow \pi_{\mathrm{CC}}{ }^{*}$ delocalization in \$CHOOSE-I (Chart 2). The $n_{\mathrm{S2}} \rightarrow \pi_{\mathrm{CC}}{ }^{*}$ stabilization energies estimated peturbatively for the two PhSNO conformers ( $20 \mathrm{kcal} / \mathrm{mol}$, Figure $9, \mathrm{~A}$ and $\mathrm{B}$ ) are comparable to a similar, but expectedly stronger, conjugation in thiophenolate anion $\mathrm{PhS}^{-}(\sim 35 \mathrm{kcal} / \mathrm{mol}$, Figure $9 \mathrm{C})$. 


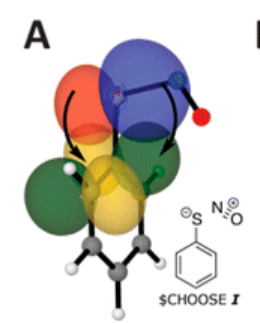

$n_{\mathrm{s} 2} \rightarrow \pi_{\mathrm{cc}}^{*}$ $\Delta E_{i j}^{(2)} \mid 21.8 \mathrm{kcal} / \mathrm{mol}$

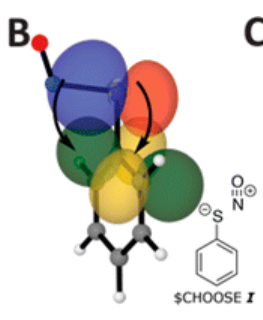

$n_{\mathrm{sz}} \rightarrow \pi_{\mathrm{cc}}^{*}$

$19.0 \mathrm{kcal} / \mathrm{mol}$

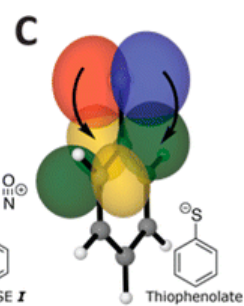

$n_{\mathrm{s}} \rightarrow \pi_{\mathrm{cc}}^{*}$

$34.5 \mathrm{kcal} / \mathrm{mol}$

Figure 9. Donor-acceptor interaction between the $n_{\mathrm{S2}}$ orbital to one of the $\pi_{\mathrm{cc}}{ }^{*}$ orbitals of the aromatic ring in $\$ C H O O S E-I$ representations of cis- (A) and trans-PhSNO (B), along with analogous conjugation in $\mathrm{PhS}^{-}(\mathrm{C})$.

The second-order $n_{\mathrm{S} 2} \rightarrow \pi_{\mathrm{cc}}{ }^{*}$ energies are at the maximum for the minimum-energy PhSNO structures with perpendicular SNO-phenyl ring arrangement $\left(\varphi=0^{\circ}\right.$, Figure 10). Thus, the secondary $n_{\mathrm{S} 2} \rightarrow \pi_{\mathrm{CC}}{ }^{*} / \sigma_{\mathrm{SN}}{ }^{*} \rightarrow$ $\pi_{\mathrm{cc}}{ }^{*}$ donor-acceptor interaction appears to be an important factor that counteracts the destabilizing steric repulsions and thus stabilizes the perpendicular PhSNO geomries (Figure 6). Furthermore, the $n_{\mathrm{S2}} \rightarrow$ $\pi_{\mathrm{cc}}{ }^{*}$ interaction energy is smaller and has a flatter profile around $\varphi=90^{\circ}$ for trans-PhSNO vs cis-PhSNO which is consistent with lower stability of the trans conformer and its flat conformational profile (Figure 4). This, in turn, correlates with weaker $n_{0} \rightarrow \sigma_{S N}{ }^{*}$ negative hyperconjugation in trans- vs cis-PhSNO (Figure 5A), the primary donor-acceptor interaction that populates the $\sigma_{S N}{ }^{*}$ orbital. Of course, the conformational profiles of PhSNO are determined by a complex interplay of a multitude of electronic and steric factors and so cannot be rationalized in their entirety in terms of just a few donor-acceptor and steric interactions. However, the long-range delocalization of the electron density from the oxygen lone pair $n_{\circ}$ to $\sigma$-antibonding $\mathrm{S}-\mathrm{N}$ orbital and then to the phenyl ring $\pi$-system appears to be an important factor determining the properties of PhSNO, as well as ArSNOs in general.

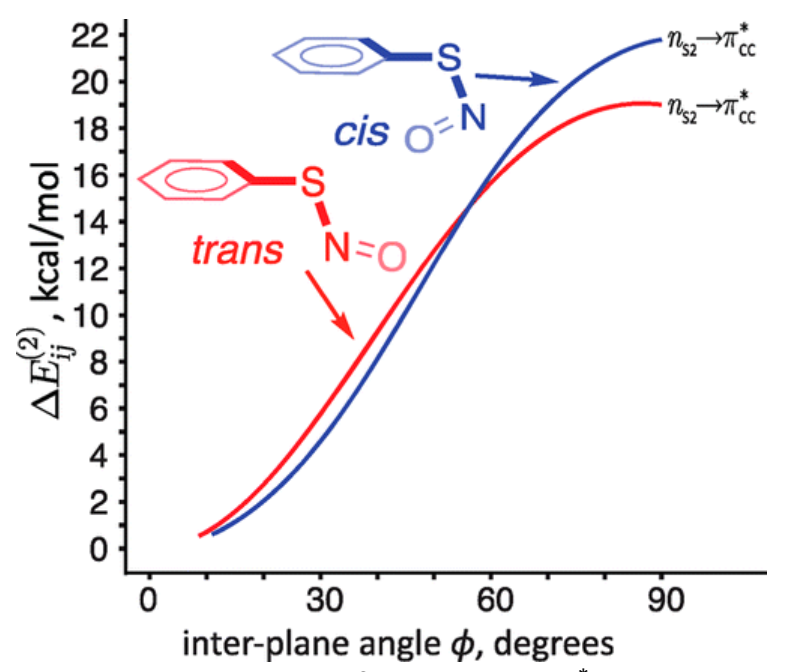

Figure 10. Evolution of the $n_{s 2} \rightarrow \pi_{c c}{ }^{*}$ interaction energies in the two PhSNO conformers with respect to the -SNO group rotation along the $\mathrm{C}-\mathrm{S}$ bond, evaluated with second-order perturbation theory in \$CHOOSEI representation. 


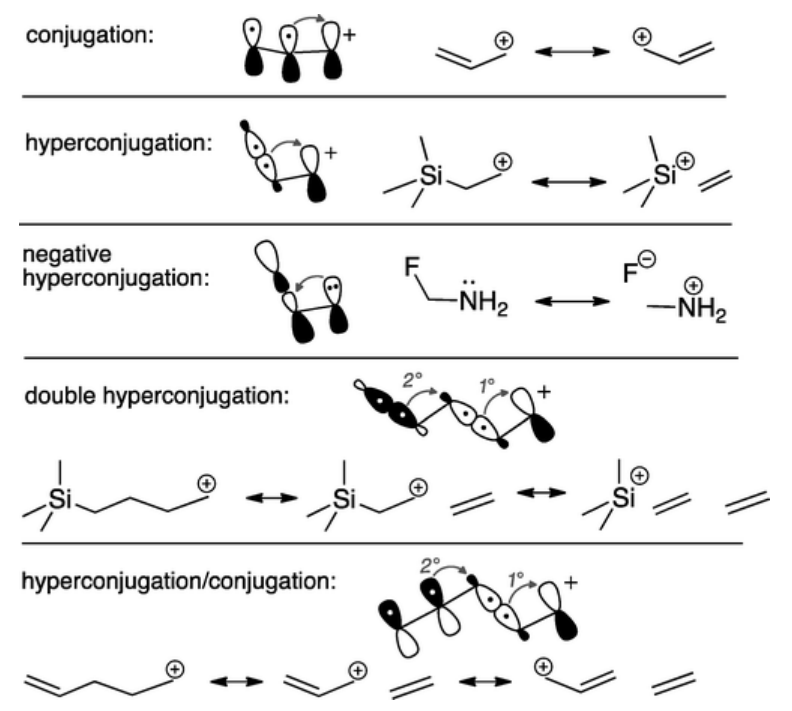

Chart 3. Basic and Extended Types of Delocalization with Examples

\section{Cascading Negative Hyperconjugation/Conjugation and Its Role in ArSNO Chemistry}

To formally characterize the cascading donor-acceptor interaction tying together the aromatic $\pi$-system and the -SNO group, we first revisit the general classification of the common donor-acceptor/conjugative orbital interactions (Chart 3). Interaction between $\pi$-orbitals and/or $p$-type lone pairs, $\pi \rightarrow \pi^{*}, n \rightarrow \pi^{*}$, etc., is referred to as conjugation or simple conjugation. $(63,64)$ Hyperconjugation refers to $\sigma \rightarrow \pi^{*}$ or $\sigma \rightarrow n$ delocalization from filled $\sigma$-orbitals into $\pi^{*}$-orbitals or empty $p$-orbitals, whereas delocalization from filled $\pi$-orbitals or $p$-type lone pairs to $\sigma^{*}$-orbitals is referred to as negative hyperconjugation.

Consecutive and cooperative donor-acceptor interactions between multiple $\pi$-orbitals are common in chemistry; e.g., they give rise to strongly coupled extended aromatic or polyene $\pi$-systems able to provide a medium for long-range charge and energy transfer.(65) On the other hand, extended/cascading donor-acceptor interactions involving orbitals other than $\pi$-type are much less common. Two- and three-stage delocalizations involving $\sigma$-orbitals have been discussed $(63,64)$ for carbocations-silicon- or tin-containing carbocations in particular. $(66,67)$ While double and triple hyperconjugations involving consecutive $\sigma \rightarrow n$ interactions, where $n$ is an empty $p$-orbital, (Chart 3) have been observed in several cases, $(63,66-68)$ a mixed case of hyperconjugation/conjugation, i.e., primary $\sigma \rightarrow \pi^{*}$ donation $\left(1^{\circ}\right)$ followed by secondary $\pi \rightarrow \pi^{*}$ conjugation $\left(2^{\circ}\right)$, has been hypothesized but not observed in real molecules.(63) Thus, extending the classification by Lambert and Ciro,(63) the cascading $n_{\mathrm{O}} \rightarrow \sigma_{\mathrm{SN}}{ }^{*}$ (primary, $\left.1^{\circ}\right) / n_{\mathrm{S2}} \rightarrow \pi_{\subset \mathrm{c}}{ }^{*}\left(\right.$ secondary, $2^{\circ}$ ) donor-acceptor interaction in PhSNO can be characterized as negative hyperconjugation/conjugation, which we will henceforth abbreviate as $n \sigma / n \pi$ (Chart 4).

\section{Chart 4}

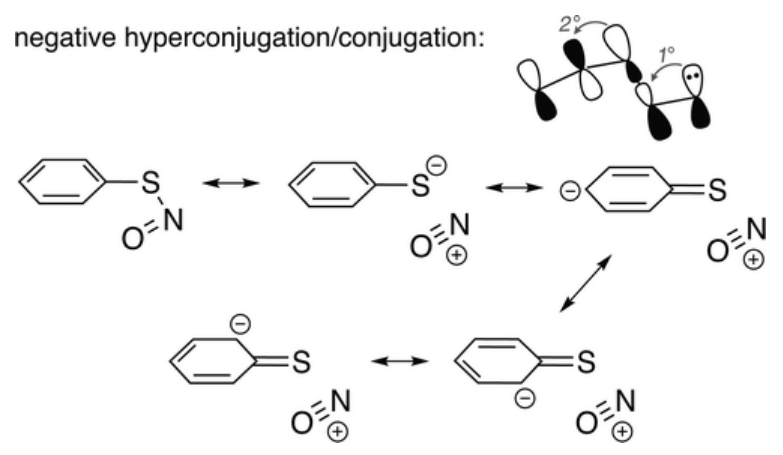


Resonance representation (Chart 4) immediately reveals the chemical consequences of the cascading $n \sigma / n \pi$ interaction in PhSNO: the secondary $n_{\mathrm{S2}} \rightarrow \pi_{\mathrm{cc}}{ }^{*}$ interaction delocalizes the formal negative charge on the sulfur atom in the ionic structure $\boldsymbol{I}$ (Chart 1), thus increasing its overall contribution into the -SNO group electronic structure. This stabilization is identical in nature to the resonance stabilization of thiophenolate anion:

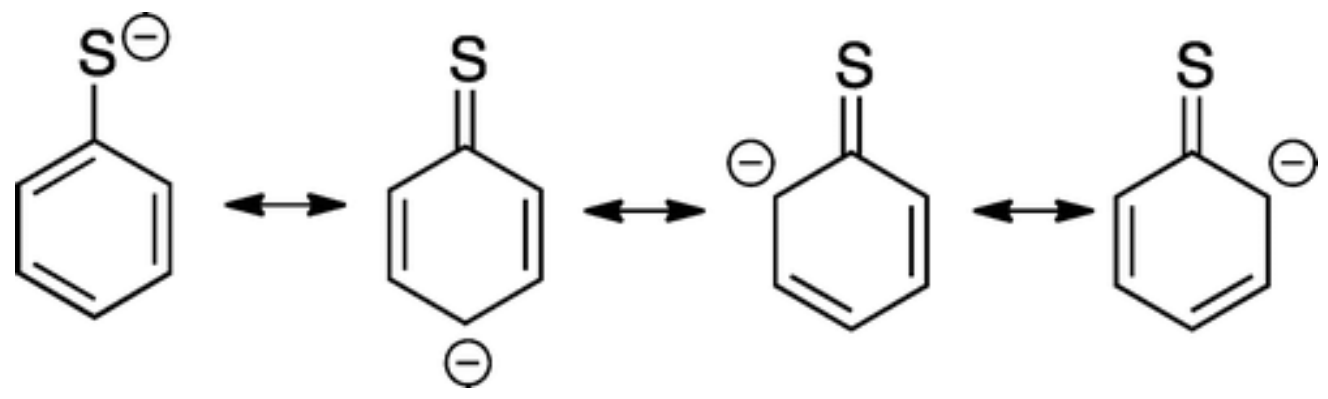

These considerations naturally explain significantly weaker $\mathrm{S}-\mathrm{N}$ bond in aromatic RSNOs, as well as the influence of the subsitituents in the aromatic ring on the properties of the -SNO group and the S-N bond in particular. Electron-withdrawing groups at para and ortho positions should further stabilize the ionic component $I$, and so result in longer and weaker $\mathrm{S}-\mathrm{N}$ bond. On the other hand, electron-donating substituents at these positions would decrease the ionic contribution and thus shorten and stabilize the $\mathrm{S}-\mathrm{N}$ bond.

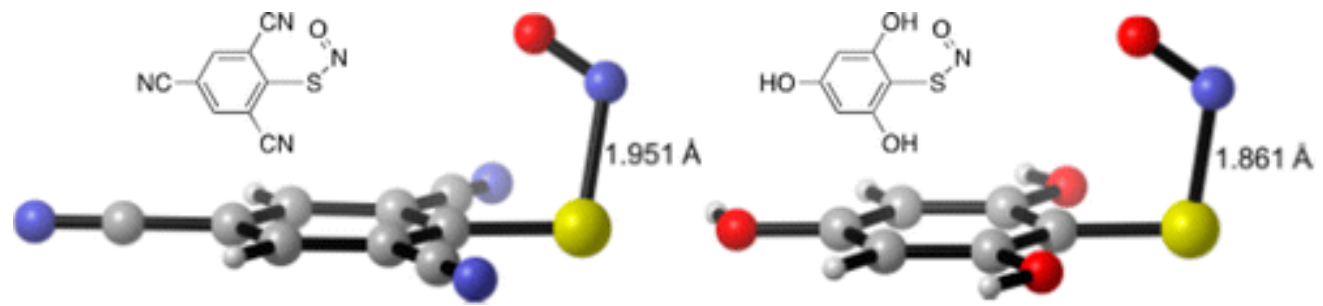

Figure 11. Subtituent effect on the S-N bond length in aromatic RSNOs.

Although a systematic investigation of the substituent effects in ArSNOs will be reported elsewhere, we show here two examples confirming this prediction (Figure 11): placing strongly electron-withdrawing $-\mathrm{CN}$ groups at ortho and para positions results in significant elongation of the $\mathrm{S}-\mathrm{N}$ bond compared to unsubstituted PhSNO (by $0.073 \AA$ ) . On the other hand, electron-donating $-\mathrm{OH}$ substituents at the same positions lead to the S-N bond shortening (by $0.017 \AA$ ). These predictions also offer a reinterpretation of the limited experimental data available. The relative stability of ArSNOs with bulky substituents reported in the literature (Figure 1) have been rationalized solely in terms of a steric protection of the -SNO group.(43-45) However, it is now clear that destabilization of the ionic resonance component of the -SNO structure due to weakly electron-donating groups at ortho-positions is another important factor responsible for the thermal stability of these ArSNOs. These examples illustrate that the electronic interaction between the aromatic $\pi$-system and the $\sigma$-orbitals of the -SNO bond, primarily in the form of the secondary $n_{\mathrm{S} 2} \rightarrow \pi_{\mathrm{cc}}{ }^{*}$ donation, is the key to understanding the substituent effects in ArSNOs.

\section{Summary}

A detailed electronic structure analysis of the parent aromatic S-nitrosothiol PhSNO reported here shows that many of the puzzling ArSNO properties can be related to a cascading negative hyperconjugation/conjugation $n \sigma / n \pi$ interaction that involves electron delocalization from the oxygen lone pair to the $\sigma$-antibonding $\mathrm{S}-\mathrm{N}$ orbital and then to the $\pi^{*}$-aromatic orbitals. To the best of our knowledge, this is the first reported example of this unusual extended delocalization pattern.

The $n \sigma / n \pi$ interaction, which can be interpreted as a stabilization of the -SNO group ionic resonance structure $I$ by the aromatic ring, leads to weakening of the $\mathrm{S}-\mathrm{N}$ bond, which correlates with lower thermal 
stability of ArSNOs vs aliphatic RSNOs. The electronic communication between the phenyl ring and the -SNO group provided by the $n \sigma / n \pi$ interaction explains the surprising substituent modulation of the $\mathrm{S}-\mathrm{N}$ bond properties in ArSNOs.

The cascading $n \sigma / n \pi$ interaction is also one of the important factors determining the perpendicular orientation of the -SNO group with respect to the aromatic plane. However, our analysis suggests that the conformational profile for the -SNO group rotation is governed by a complex interplay of steric repulsions, donor-acceptor delocalizations, and geometry distortions. While cis-PhSNO has a clear preference for perpendicular SNO-phenyl ring orientation, in trans-PhSNO the -SNO tilt angle would be evenly distributed within $60^{\circ}\left(90^{\circ} \pm 30^{\circ}\right.$, Figure 4) at finite temperatures. At the same time, the experimental $(43,44)$ and computational $(47)$ results from the literature suggest that substituted ArSNOs can have a titled -SNO group orientation both in cis and trans conformers. Further studies will be required to discern the combination of factors determining the variation of the -SNO group tilt in ArSNOs.

The present results lay a solid conceptual groundwork for further investigation of the substituted aromatic RSNOs and design of novel RSNO-based $\mathrm{NO}, \mathrm{NO}^{+}$, and $\mathrm{HNO} / \mathrm{NO}^{-}$donors. Also, this work once again illustrates that the antagonistic resonance description of the -SNO group (Chart 1) provides a powerful paradigm to understand and predict chemistry of RSNOs, including their structure, stability, reactivity, and the biological control of their reactions.

\section{Supporting Information}

Figures S1 and S2, complete citation for ref 51, Cartesian coordinates, and energies of the calculated structures. This material is available free of charge via the Internet at http://pubs.acs.org.

\section{Terms \& Conditions}

Electronic Supporting Information files are available without a subscription to ACS Web Editions. The American Chemical Society holds a copyright ownership interest in any copyrightable Supporting Information. Files available from the ACS website may be downloaded for personal use only. Users are not otherwise permitted to reproduce, republish, redistribute, or sell any Supporting Information from the ACS website, either in whole or in part, in either machine-readable form or any other form without permission from the American Chemical Society. For permission to reproduce, republish and redistribute this material, requesters must process their own requests via the RightsLink permission system. Information about how to use the RightsLink permission system can be found at http://pubs.acs.org/page/copyright/permissions.html.

\section{Acknowledgment}

Q.K.T. is a recipient of the National Science Foundation (NSF) CAREER award CHE-1255641, and Marquette University Way-Klinger Young Scholar Award. This work used the high-performance computing cluster Père at Marquette University funded by NSF Awards OCI-0923037 and CBET-0521602, and the Extreme Science and Engineering Discovery Environment (XSEDE) supported by NSF Grant ACl-1053575.

\section{References}

1 Williams, D. L. H. The Chemistry of S-Nitrosothiols Acc. Chem. Res. 1999, 32, 869- 876

2 Broniowska, K. A.; Hogg, N. The Chemical Biology of S-Nitrosothiols Antioxid. Redox Signaling 2012, 17, 969- 980

3 Smith, B. C.; Marletta, M. A. Mechanisms of S-Nitrosothiol Formation and Selectivity in Nitric Oxide Signaling Curr. Opin. Chem. Biol. 2012, 16, 498- 506 
4 Koppenol, W. H. Nitrosation, Thiols, and Hemoglobin: Energetics and Kinetics Inorg.

Chem. 2012, 51, 5637- 5641

5 Seth, D.; Stamler, J. S. The SNO-proteome: causation and classifications Curr. Opin. Chem.

Biol. 2011, 15, 129-136

6 Hess, D. T.; Matsumoto, A.; Kim, S. O.; Marshall, H. E.; Stamler, J. S. Protein S-Nitrosylation: Purview and Parameters Nat. Rev. Mol. Cell Biol. 2005, 6, 150- 166

7 Doulias, P.-T.; Greene, J. L.; Greco, T. M.; Tenopoulou, M.; Seeholzer, S. H.; Dunbrack, R. L.; Ischiropoulos, $\mathrm{H}$. Structural profiling of endogenous S-nitrosocysteine residues reveals unique features that accommodate diverse mechanisms for protein S-nitrosylation Proc. Natl. Acad. Sci.

U.S.A. 2010, 107, 16958- 16963

8 Wong, P. S.; Hyun, J.; Fukuto, J. M.; Shirota, F. N.; DeMaster, E. G.; Shoeman, D. W.; Nagasawa, H. T. Reaction Between S-Nitrosothiols and Thiols: Generation of Nitroxyl (HNO) and Subsequent

Chemistry Biochemistry 1998, 37, 5362- 5371

9 Heinrich, T. A.; da Silva, R. S.; Miranda, K. M.; Switzer, C. H.; Wink, D. A.; Fukuto, J. M. Biological nitric oxide signalling: chemistry and terminology Br. J. Pharmacol. 2013, 169, 1417-1429

10 Flores-Santana, W.; Salmon, D. J.; Donzelli, S.; Switzer, C. H.; Basudhar, D.; Ridnour, L.; Cheng, R.; Glynn, S. A.; Paolocci, N.; Fukuto, J. M.; Miranda, K. M.; Wink, D. A. The Specificity of Nitroxyl Chemistry is Unique Among Nitrogen Oxides in Biological Systems Antioxid. Redox Signaling 2011, 14, 1659- 1674

11 Filipovic, M. R.; Eberhardt, M.; Prokopovic, V.; Mijuskovic, A.; Orescanin-Dusic, Z.; Reeh, P.; IvanovicBurmazovic, I. Beyond $\mathrm{H}_{2} \mathrm{~S}$ and NO Interplay: Hydrogen Sulfide and Nitroprusside React Directly to Give Nitroxyl (HNO). A New Pharmacological Source of HNO J. Med. Chem. 2013, 56, 1499- 1508

12 Filipovic, M. R.; Miljkovic, J. L. j.; Nauser, T.; Royzen, M.; Klos, K.; Shubina, T.; Koppenol, W. H.; Lippard, S. J.; Ivanović-Burmazović, I. Chemical Characterization of the Smallest S-Nitrosothiol, HSNO; Cellular Cross-talk of $\mathrm{H}_{2} \mathrm{~S}$ and S-Nitrosothiols J. Am. Chem. Soc. 2012, 134, 12016- 12027

13 Miljkovic, J. L. j.; Kenkel, I.; Ivanović-Burmazović, I.; Filipovic, M. R. Generation of HNO and HSNO from Nitrite by Heme-Iron-Catalyzed Metabolism with $\mathrm{H}_{2} \mathrm{~S}$ Angew. Chem., Int. Ed. Engl. 2013, 52, 12061- 12064

$14 \mathrm{King}$, S. B. Potential biological chemistry of hydrogen sulfide $\left(\mathrm{H}_{2} \mathrm{~S}\right)$ with the nitrogen oxides Free Radical Biol. Med. 2013, 55, 1- 7

15 Broniowska, K. A.; Diers, A. R.; Hogg, N. S-Nitrosoglutathione Biochim. Biophys. Acta 2013, 1830, 3173- 3181

16 Scatena, R.; Bottoni, P.; Pontoglio, A.; Giardina, B. Pharmacological Modulation of Nitric Oxide Release: New Pharmacological Perspectives, Potential Benefits and Risks Curr. Med. Chem. 2010, 17, 61- 73

17 Al-Sa'doni, H. H.; Ferro, A. Current Status and Future Possibilities of Nitric Oxide-Donor Drugs: Focus on SNitrosothiols Mini-Rev. Med. Chem. 2005, 5, 247- 254

18 Scatena, R.; Bottoni, P.; Martorana, G. E.; Giardina, B. Nitric oxide donor drugs: an update on pathophysiology and therapeutic potential Expert Opin. Invest. Drugs 2005, 14, 835- 846

19 Gaucher, C.; Boudier, A.; Dahboul, F.; Parent, M.; Leroy, P. S-nitrosation/denitrosation in Cardiovascular Pathologies: Facts and Concepts for the Rational Design of S-Nitrosothiols Curr. Pharm.

Des. 2013, 19, 458-472

20 Parent, M.; Boudier, A.; Dupuis, F.; Nouvel, C.; Sapin, A.; Lartaud, I.; Six, J. L.; Leroy, P.; Maincent, P. Are in situ formulations the keys for the therapeutic future of S-nitrosothiols? Eur. J. Pharm.

Biopharm. 2013, 85, 640-649

21 Pegalajar-Jurado, A.; Joslin, J. M.; Hawker, M. J.; Reynolds, M. M.; Fisher, E. R. Creation of Hydrophilic Nitric Oxide Releasing Polymers via Plasma Surface Modification ACS Appl. Mater.

Interfaces 2014, 6, 12307- 12320

22 Baciu, C.; Gauld, J. W. An Assessment of Theoretical Methods for the Calculation of Accurate Structures and S-N Bond Dissociation Energies of S-Nitrosothiols (RSNOs) J. Phys. Chem. A 2003, 107, 9946- 9952

$23 \mathrm{Fu}$, Y.; Mou, Y.; Lin, B.-L.; Liu, L.; Guo, Q.-X. Structures of the XY-NO Molecules and Homolytic Dissociation Energies of the Y-NO bonds ( $Y=$ C, N, O, S) J. Phys. Chem. A 2002, 106, 12386- 12392 
24 Bartberger, M. D.; Mannion, J. D.; Powell, S. C.; Stamler, J. S.; Houk, K. N.; Toone, E. J. SN Dissociation Energies of S-Nitrosothiols: On the Origins of Nitrosothiol Decomposition Rates J. Am. Chem. Soc. 2001, 123, 8868-8869

25 Ivanova, L. V.; Anton, B. J.; Timerghazin, Q. K. On the possible biological relevance of HSNO isomers: a computational investigation Phys. Chem. Chem. Phys. 2014, 16, 8476-8486

26 Méndez, M.; Francisco, J. S.; Dixon, D. A. Thermodynamic Properties of the Isomers of [HNOS], $\left[\mathrm{HNO}_{2} \mathrm{~S}\right]$, and $\left[\mathrm{HNOS}_{2}\right]$ and the Role of the Central Sulfur Chem.-Eur. J. 2014, 20, 10231- 10235

27 Arulsamy, N.; Bohle, D. S.; Butt, J. A.; Irvine, G. J.; Jordan, P. A.; Sagan, E. Interrelationships between Conformational Dynamics and the Redox Chemistry of S-Nitrosothiols J. Am. Chem. Soc. 1999, 121, 7115-7123

28 Bartberger, M. D.; Houk, K. N.; Powell, S. C.; Mannion, J. D.; Lo, K. Y.; Stamler, J. S.; Toone, E. J. Theory, Spectroscopy, and Crystallographic Analysis of S-Nitrosothiols: Conformational Distribution Dictates Spectroscopic Behavior J. Am. Chem. Soc. 2000, 122, 5889- 5890

29 Toubin, C.; Yeung, D. Y.; English, A. M.; Peslherbe, G. H. Theoretical Evidence that Cu' Complexation Promotes Degradation of S-Nitrosothiols J. Am. Chem. Soc. 2002, 124, 14816- 14817

30 Baciu, C.; Cho, K. B.; Gauld, J. W. Influence of $\mathrm{Cu}^{+}$on the RS-NO Bond Dissociation Energy of S-Nitrosothiols J. Phys. Chem. B 2005, 109, 1334- 1336

31 Perissinotti, L. L.; Estrin, D. A.; Leitus, G.; Doctorovich, F. A Surprisingly Stable S-Nitrosothiol Complex J. Am. Chem. Soc. 2006, 128, 2512- 2513

32 Perissinotti, L. L.; Leitus, G.; Shimon, L.; Estrin, D.; Doctorovich, F. A Unique Family of Stable and WaterSoluble S-Nitrosothiol Complexes Inorg. Chem. 2008, 47, 4723- 4733

33 Zeida, A.; Guardia, C. M.; Lichtig, P.; Perissinotti, L. L.; Defelipe, L. A.; Turjanski, A.; Radi, R.; Trujillo, M.; Estrin, D. A. Thiol redox biochemistry: insights from computer simulations Biophy. Rev. 2014, 6, 27- 46

34 Timerghazin, Q. K.; Talipov, M. R. Unprecedented External Electric Field Effects on S-Nitrosothiols: Possible Mechanism of Biological Regulation? J. Phys. Chem. Lett. 2013, 4, 1034-1038

35 Timerghazin, Q. K.; Peslherbe, G. H.; English, A. M. Resonance Description of S-nitrosothiols: Insights into Reactivity Org. Lett. 2007, 9, 3049- 3052

36 Talipov, M. R.; Timerghazin, Q. K. Protein Control of S-Nitrosothiol Reactivity: Interplay of Antagonistic Resonance Structures J. Phys. Chem. B 2013, 117, 1827- 1837

37 Talipov, M. R.; Khomyakov, D. G.; Xian, M.; Timerghazin, Q. K. Computational Design of S-Nitrosothiol "Click" Reactions J. Comput. Chem. 2013, 34, 1527- 1530

38 Timerghazin, Q. K.; Peslherbe, G. H.; English, A. M. Structure and stability of HSNO, the simplest Snitrosothiol Phys. Chem. Chem. Phys. 2008, 10, 1532- 1539

39 Timerghazin, Q. K.; English, A. M.; Peslherbe, G. H. On the multireference character of S-nitrosothiols: A theoretical study of HSNO Chem. Phys. Lett. 2008, 454, 24- 29

40 Tasker, H. S.; Jones, H. O. CCXII. The Action of Mercaptans on Acid Chlorides. Part II. The Acid Chlorides of Phosphorous, Sulphur, and Nitrogen J. Chem. Soc., Trans. 1909, 95, 1910- 1918

41 Oae, S.; Fukushima, D.; Kim, Y. H. Novel Method of Activating Thiols by their Conversion into Thionitrites with Dinitrogen Tetroxide J. Chem. Soc., Chem. Commun. 1977, 407b- 4408

42 Oae, S.; Kim, Y. H.; Fukushima, D.; Shinhama, K. New Syntheses of Thionitrites and their Chemical Reactivites J. Chem. Soc., Perkin Trans. 1 1978, 913- 917

43 Goto, K.; Hino, Y.; Takahashi, Y.; Kawashima, T.; Yamamoto, G.; Takagi, N.; Nagase, S. Synthesis, Structure, and Reactions of the First Stable Aromatic S-Nitrosothiol Bearing a Novel Dendrimer-Type Steric Protection Group Chem. Lett. 2001, 30, 1204-1205

44 Itoh, M.; Takenaka, K.; Okazaki, R.; Takeda, N.; Tokitoh, N. The First Stable Aromatic S-nitrosothiol: Synthesis, Structure and Reactivity Chem. Lett. 2001, 1206-1207

45 Grossi, L.; Montevecchi, P. C. A Kinetic Study of S-Nitrosothiol Decomposition Chem.-Eur. J. 2002, 8, 380- 387

46 Petit, C.; Hoffmann, P.; Souchard, J.-P.; Labidalle, S. Synthesis and Characterization of New Aromatic Thionitrites Phosphorus, Sulfur Silicon Relat. Elem. 1997, 129, 59- 67 
47 Marazzi, M.; López-Delgado, A.; Fernández-González, M. A.; Castaño, O.; Frutos, L. M.; Temprado, M. Modulating Nitric Oxide Release by S-Nitrosothiol Photocleavage: Mechanism and Substituent Effects J. Phys. Chem. A 2012, 116, 7039- 7049

48 Weinhold, F.; Landis, C. R. Discovering Chemistry with Natural Bond Orbitals; Wiley: Hoboken, NJ, 2012.

49 Perdew, J. P.; Burke, K.; Ernzerhof, M. Generalized Gradient Approximation Made Simple Phys. Rev. Lett. 1996, 77, 3865

50 Adamo, C.; Barone, V. Toward reliable density functional methods without adjustable parameters: The PBEO model J. Chem. Phys. 1999, 110, 6158

51 Frisch, M. J.;Gaussian 09, revision D.01; Gaussian, Inc.: Wallingford, CT, 2009.

52 Jensen, F. Polarization Consistent Basis Sets: Principles J. Chem. Phys. 2001, 115, 9113- 9125

53 Jensen, F. Polarization consistent basis sets. III. The importance of diffuse functions J. Chem. Phys. 2002, 117, 9234

54 Schuchardt, K. L.; Didier, B. T.; Elsethagen, T.; Sun, L.; Gurumoorthi, V.; Chase, J.; Li, J.; Windus, T. L. Basis Set Exchange: A Community Database for Computational Sciences J. Chem. Inf. Model. 2007, 47, 1045- 1052

55 Peng, C.; Ayala, P. Y.; Schlegel, H. B.; Frisch, M. J. Using Redundant Internal Coordinates to Optimize Equilibrium Geometries and Transition States J. Comput. Chem. 1996, 17, 49- 56

56 Glendening, E. D.; Badenhoop, J.; Reed, A. E.; Carpenter, J. E.; Bohmann, J. A.; Morales, C. M.; Weinhold, F.; Landis, C. R.NBO 6.0; Theoretical Chemistry Institute, University of Wisconsin: Madison, WI, 2013.

57 Badenhoop, J. K.; Weinhold, F. Natural Bond Orbital Analysis of Steric Interactions J. Chem. Phys. 1997, 107, 5406

58 Lü, J.-M.; Wittbrodt, J. M.; Wang, K.; Wen, Z.; Schlegel, H. B.; Wang, P. G.; Cheng, J.-P. NO Affinities of SNitrosothiols: A Direct Experimental and Computational Investigation of RS-NO Bond Dissociation Energies J. Am. Chem. Soc. 2001, 123, 2903- 2904

59 Nyburg, S. C. Bond order-bond length correlations for SN bonds in organic molecules J. Chem. Crystallogr. 1973, 3, 331- 336

60 Bharatam, P. V. Negative hyperconjugative interactions in S-nitrosothiols: a theoretical study Tetrahedron Lett. 2002, 43, 8289-8291

61 Jakobsche, C. E.; Choudhary, A.; Miller, S. J.; Raines, R. T. $n \rightarrow \pi^{*}$ Interaction and $\left.n\right)(\pi$ Pauli Repulsion are Antagonistic for Protein Stability J. Am. Chem. Soc. 2010, 132, 6651- 6653

62 The latest NBO version, NBO 6.0, does not calculate any donor-acceptor interactions between weakly occupied non-Lewis orbitals; NBO 5.9 does not report these interactions in the second-order perturbation analysis section, although it still detects delocalizations from non-Lewis orbitals with the second-order approach and lists them in the NBO Summary section. Legacy NBO 3.1 code prints some of these interactions in the second-order perturbation analysis section, although the reported interaction energies are unphysical and should be disregarded.

63 Lambert, J. B.; Ciro, S. M. The Interaction of $\pi$ Orbitals with a Carbocation over Three $\sigma$ Bonds J. Org. Chem. 1996, 61, 1940-1945

64 Alabugin, I. V.; Gilmore, K. M.; Peterson, P. W. Hyperconjugation WIREs Comput. Mol. Sci. 2011, 1, 109- 141 65 Milián-Medina, B.; Gierschner, J. $\pi$-Conjugation WIREs Comput. Mol. Sci. 2012, 2, 513- 524

$66 \mathrm{Grob}, \mathrm{C}$. A.; Rich, R. Inductive and Hyperconjugative Effects in the Solvolysis of 4-substituted Bicyclo [2.2.2] Oct-1-yl p-Nitrobenzenesulfonates Tetrahedron Lett. 1978, 19, 663- 666

67 Adcock, W.; Coope, J.; Shiner, V. J., Jr; Trout, N. A. Evidence for 2-fold Hyperconjugation in the Solvolysis of 5(Trimethylsilyl) and 5-(Trimethylstannyl)-2-adamantyl Sulfonates J. Org. Chem. 1990, 55, 1411- 1412

68 Alabugin, I. V.; Manoharan, M. Effect of Double-Hyperconjugation on the Apparent Donor Ability of SigmaBonds: Insights from the Relative Stability of Delta-Substituted Cyclohexyl Cations J. Org.

Chem. 2004, 69, 9011-9024 